\title{
A remark on R. Moeckel's paper 'Geodesics on modular surfaces and continued fractions'
}

\author{
TOSHIHIRO NAKANISHI \\ Department of Mathematics, Shizuoka University, Shizuoka 422, Japan
}

(Received 23 February 1987 and revised 10 March 1989)

Abstract. It is shown that a result by Moeckel holds not only for admissible subgroups of $\operatorname{SL}(2, \mathbb{Z})$, but also for arbitrary subgroups of finite index.

The modular group $\Gamma=\operatorname{SL}(2, \mathbb{Z})$ acts discontinuously on a hyperbolic plane $\mathscr{H}=\{z=x+\mathrm{i} y ; y>0\}$. Let $G$ be a subgroup of finite index in $\Gamma$. In his paper [1] Moeckel obtained the following result [1, Proposition 2.1]:

Let $C$ be a $G$-cusp. If $G$ is admissible, then for almost every irrational number $\beta$,

$$
\lim _{N \rightarrow \infty} \frac{1}{N}\left|\left\{n \leq N ; \beta_{n} \in C\right\}\right|=w(C) /[\Gamma: G],
$$

where $\beta_{n}$ is the $n$th approximant $\left[b_{0}, b_{1}, \ldots, b_{n}\right]$ of the continued fraction espansion of $\beta=\left[b_{0}, b_{1}, \ldots,\right]$, and $w(C)$ denotes the width of $C$.

The objective of the present note is to show that Moeckel's proposition holds without the assumption that $G$ is admissible.

It is necessary to say a few words about the correct statement of our generalization of Moeckel's proposition. Let $\bar{G}$ be the inhomogenized group of $G$ in $\bar{\Gamma}=\operatorname{PSL}(2, \mathbb{Z})$ ([2, p. 71]). As $\Gamma$ is actually viewed as a group of linear fractional transformations, the statement of the generalized proposition is the same as Moeckel's, but (1) is replaced by

$$
\lim _{N \rightarrow \infty} \frac{1}{N}\left|\left\{n \leq N ; \beta_{n} \in C\right\}\right|=w(C) /[\bar{\Gamma}: \bar{G}] .
$$

Here $2[\Gamma: G]=[\bar{\Gamma}: \bar{G}]$ if $-\mathbf{I} \notin G$ and $[\Gamma: G]=[\bar{\Gamma}: \bar{G}]$ if $-\mathbf{I} \in G$, where $\mathbf{I}$ is the unit matrix.

Let 2 be the fundamental quadrilateral defined by

$$
\mathscr{Q}=\{z=x+\mathrm{i} y ; 0 \leq x<1,|z| \geq 1,|z-1|>1\} \cup\{(1+\sqrt{3} \mathrm{i}) / 2\} .
$$

Let $S(z)=1 /(-z+1)$. An elementary triangle is the image of $\mathscr{Q} \cup S \mathscr{Q} \cup S^{2} \mathscr{Q}$ under an element of $\Gamma$. The group $G$ partitions the rational numbers into equivalence classes called G-cusps. 
Let $C$ be a $G$-cusp and $\Delta$ be an elementary triangle with a vertex in $C$. The triangle $\Delta$ may be left invariant by a cyclic subgroup of order 3 in $G$, which does not occur for admissible groups. Hence the restriction of the canonical projection $\pi: \mathscr{H} \rightarrow \mathscr{H} / G$ to $\Delta$ may fail to be injective. To establish Moeckel's proposition we need a function on $T_{1}(\mathscr{H} / G)$, the unit tangent bundle to $\mathscr{H} / G$, of the same character as the function $f_{\left(C, \Delta^{\prime}\right)}$ in the proof of Proposition 3.2 of [1]. So we define for $\Delta^{\prime}=\pi \Delta$ :

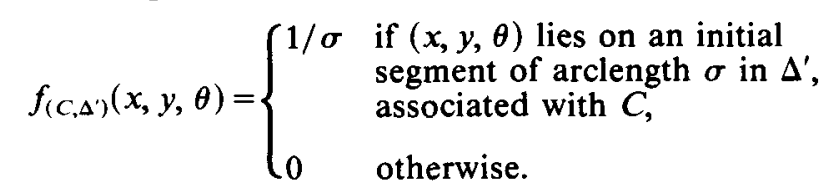

Here we view the coordinates $(x, y, \theta)$ of $T_{1} \mathscr{H}$, the unit tangent bundle to $\mathscr{H}[1$, p. 70] as local coordinates of $T_{1}(\mathscr{H} / G)$ except possibly for the fixed point of the cyclic group or order 3 . We can neglect this point for our purpose. By replacing $G$ by a conjugation of $G$ in $\Gamma$, if necessary, we can assume that $\Delta=\mathscr{2} \cup S \mathscr{Q} \cup S^{2} \mathscr{Q}$ and $\infty$ belongs to $C$. In this case the width $w(C)$ of $C$ is the smallest positive integer $k$ such that the translation $z \rightarrow z+k$, is an element of $G$, and $\Delta$ is left invariant by the cyclic group $\left\{\mathbf{I}, S, S^{2}\right\}$. Let $(x, y, \theta)$ be a point of $T_{1} \mathscr{H}$ lying on an initial segment in $\Delta$, associated with $\infty$. If we express this point by the coordinates $(\alpha, \beta, s)$ introduced at p. 70 of [1], then $-1 \leq \alpha<0$ and $1<\beta<\infty$. If $(x, y) \in 2$, then $(a, \beta)$ is in the set $\bigcup_{i=1}^{4} \Omega_{i}$ depicted in figure 1. If $(x, y) \in S \mathscr{2}$, then $(\alpha, \beta)$ is in $\Omega_{3} \cup \Omega_{4} \cup \Omega_{5}$ and if $(x, y) \in S^{2} \mathscr{Q}$, then $(\alpha, \beta)$ is in $\Omega_{2} \cup \Omega_{4} \cup \Omega_{5}$. For the case where $S$ is an element of $G$, we need the following lemma.

Lemma. Let $\left(x_{1}, y_{1}, \theta_{1}\right)$ and $\left(x_{2}, y_{2}, \theta_{2}\right)$ be points each of which lies on an initial segment in $\Delta$ associated with $\infty$. If they are equivalent under the action of $\left\{I, S, S^{2}\right\}$, then $\left(x_{1}, y_{1}, \theta_{1}\right)=\left(x_{2}, y_{2}, \theta_{2}\right)$.

Proof. Assume that the two points $\left(x_{1}, y_{1}, \theta_{1}\right)$ and $\left(x_{2}, y_{2}, \theta_{2}\right)$ are distinct. It suffices to consider the cases (1) $\left(x_{1}, y_{1}\right) \in \mathscr{Q}$ and $\left(x_{2}, y_{2}\right) \in S \mathscr{Q},(2)\left(x_{1}, y_{1}\right) \in \mathscr{Q}$ and $\left(x_{2}, y_{2}\right) \in S^{2} \mathscr{Q}$, and $(3)\left(x_{1}, y_{1}\right) \in S \mathscr{Q}$ and $\left(x_{2}, y_{2}\right) \in S^{2} \mathscr{Q}$. Express the points $\left(x_{i}, y_{i}, \theta_{i}\right)$, $i=1,2$, as $\left(\alpha_{i}, \beta_{i}, s_{i}\right)$ with $-1 \leq \alpha_{i}<0,1<\beta_{i}<\infty$. For the case (1), if the two points are equivalent under the action of $\left\{I, S, S^{2}\right\}$, then $\left(\alpha_{1}, \beta_{1}\right)=\left(S^{2} \alpha_{2}, S^{2} \beta_{2}\right)$. However, as figure 1 shows, this is impossible. The figure also shows that other cases are impossible.

It follows from the lemma that, even though $\left.\pi\right|_{\Delta}$ is not injective, the tangent vectors of $T_{1} \mathscr{H}$ lying on initial segments in $\Delta$, associated with $\infty$ and the tangent vectors of $T_{1}(\mathscr{H} / G)$ lying on initial segments in $\Delta^{\prime}$, associated with $C$ are in one-to-one correspondence. Hence for the present function $f_{\left(C, \Delta^{\prime}\right)}$ the following computation is also true $[1, p .82]$ :

$$
\begin{aligned}
\frac{1}{2} \int_{T_{1}(\mathscr{H} / G)} f_{\left(C, \Delta^{\prime}\right)} d \mu & =\int_{1}^{\infty} d \beta \int_{-1}^{0} \frac{2 d \alpha}{(\alpha-\beta)^{2}} \int_{\gamma(\alpha, \beta)} \frac{1}{\sigma} d s \\
& =2 \ln 2 .
\end{aligned}
$$

The function $f_{\left(C, \Delta^{\prime}\right)}$ is defined so that its integral over a geodesic counts the number of initial segments along the geodesic which lie in $\Delta^{\prime}$, are associated with $C$, like 


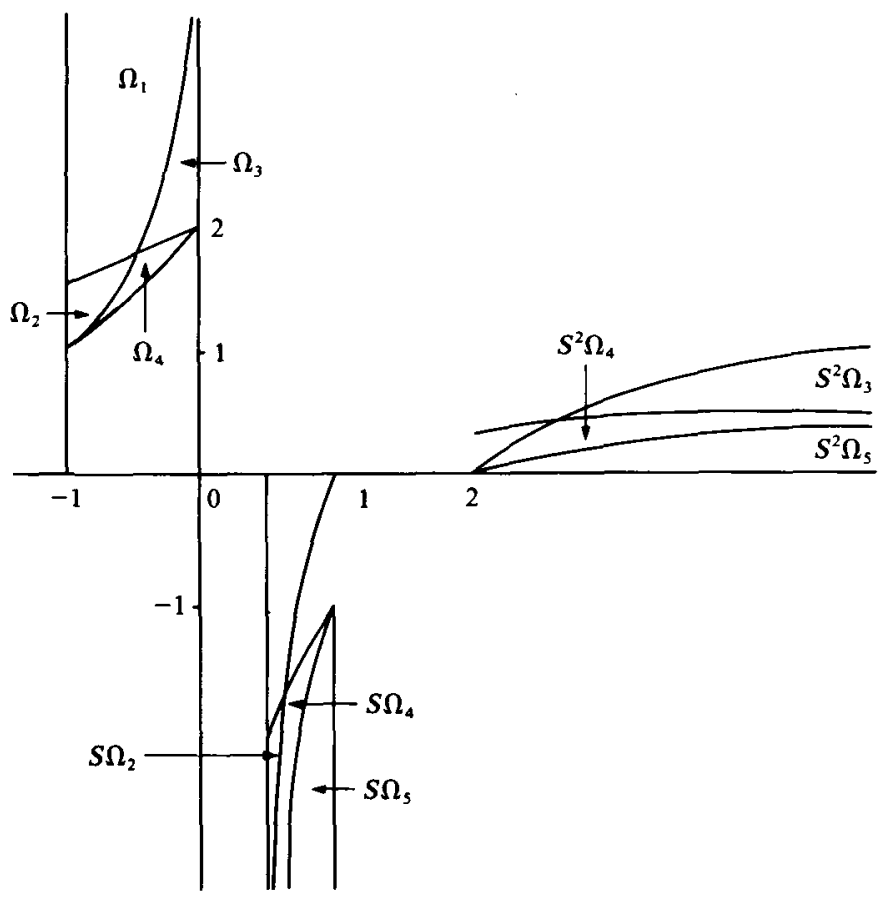

FIGURE 1

$$
\begin{aligned}
& \Omega_{1}=\{(\alpha, \beta) ; \beta \geq \max (-1 / \alpha,(2-\alpha) /(1-\alpha)\}, \\
& \Omega_{2}=\{(\alpha, \beta) ; \beta \geq-1 / \alpha, \beta<(2-\alpha) /(1-\alpha)\}, \\
& \Omega_{3}=\{(\alpha, \beta) ; \beta<-1 / \alpha, \beta \geq(2-\alpha) /(1-\alpha)\}, \\
& \Omega_{4}=\{(\alpha, \beta) ; \beta<\min (-1 / \alpha,(2-\alpha) /(1-\alpha)), \beta>(2-\alpha) /(1-2 \alpha)\}, \\
& \Omega_{5}=\{(\alpha, \beta) ; \beta \leq(2-\alpha) /(1-2 \alpha)\} .
\end{aligned}
$$

the function in Proposition 3.2 of [1]. Hence, by proceeding with this function, we can prove Proposition 3.2 for $G$ which may not be admissible. Then Moeckel's Proposition 2.1 follows, because Proposition 3.2 is a rephrasing of Proposition 2.1 in terms of the symbolic description of geodesics on $\mathscr{H} / G$.

We conclude this note by offering some examples. Let

$$
\begin{aligned}
& \Gamma_{0}(p)=\left\{\left[\begin{array}{ll}
a & b \\
c & d
\end{array}\right] \in \Gamma ; c \equiv 0 \bmod p\right\}, \quad \text { and } \\
& \Gamma^{0}(p)=\left\{\left[\begin{array}{ll}
a & b \\
c & d
\end{array}\right] \in \Gamma ; b \equiv 0 \bmod p\right\},
\end{aligned}
$$

where $p$ is a prime [2, Chap. IV, 3]. These groups are not admissible if, for example, $p=7$ and 13. But now we can apply Moeckel's Proposition to them and obtain for almost every irrational number $\beta$,

$$
\begin{aligned}
\lim _{N \rightarrow \infty} \frac{1}{N}\left|\left\{n \leq N ; p \mid P_{n}\right\}\right| & =\lim _{N \rightarrow \infty} \frac{1}{N}\left|\left\{n \leq N ; p \mid Q_{n}\right\}\right| \\
& =(1+p)^{-1},
\end{aligned}
$$


where the $n$th approximant of the continued fraction expansion of $\beta$ is presented by a reduced ratio $P_{n} / Q_{n}$ of integers.

\section{REFERENCES}

[1] R. Moeckel. Geodesics on modular surfaces and continued fractions. Ergod. Th. \& Dynam. Sys. 2 (1982), 69-83.

[2] B. Schoeneberg. Elliptic Modular Functions. Springer: Berlin-Heidelberg-New York, 1974. 